\title{
AUTOMATIC ACTIVITY SEGMENTATION FROM SURVEILLANCE VIDEO USING CONVENTIONAL SHOT BOUNDARY DETECTION METHODS
}

\author{
ANGADI S.A. ${ }^{1}$, VILAS NAIK² AND ASHWIN KUMAR ${ }^{3}$ \\ Department of Computer science and Engineering, Basaveshwar Engineering College, Bagalkot, Karnataka, India.

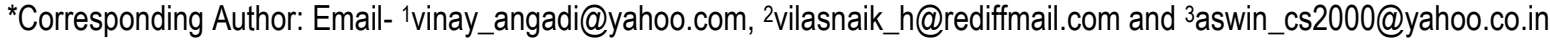

Received: April 12, 2012; Accepted: May 15, 2012

\begin{abstract}
Video-surveillance systems are one of the main source of information during investigations. However, the adopted closed-circuit devices are often affected by poor quality mainly because of economical and practical problems. In many cases a low quality image can give useful information during the first phase of the investigation. As a consequence, the images and sequences coming from video-surveillance systems need to be digitalized in order to be processed to enhance and extract features useful for crime analysis. Visual information is the most appealing and intuitive mode of conveying information. Further, the amount of information that video carries is significantly greater than that carried by any other media. From surveillance for security, to capturing and analyzing medical procedures, to forensic applications. The greatest users of video information constitute the surveillance cameras used for security. The content in such applications constitutes hours of video usually taken from a single camera that is stationary. For such applications, it is usually necessary to trace certain anomalous events or individuals .In particular, airport surveillance cameras, surveillance camera in traffic, shopping malls are very good examples. The purpose of these cameras is to detect suspicious activity, like a perpetrator leaving a piece of luggage that might be harmful, or picking up something that might not belong to him or her, Firing from a vehicle, suspicious activity in shopping malls etc.

In this work, the task is the automatic segmentation of continuous video into activity-based segments. It is different from the conventional shot-detection and scene change problems of video processing. The information being searched for is not the boundary between two different video contents in a single video, but a significant change in the activity within a single scene in the video. Thus, a temporal segmentation of the video into different events is desired. The approach used is to combine some of the conventional shot-detection methods with some new techniques for video-content analysis .
\end{abstract}

Keywords- Video-surveillance systems, crime analysis, automatic segmentation, activity-based segments, video-content analysis.

Citation: Angadi S.A., Vilas Naik and Ashwin Kumar (2012) Automatic Activity Segmentation from Surveillance Video Using Conventional Shot Boundary Detection Methods. International Journal of Machine Intelligence, ISSN: 0975-2927 \& E-ISSN: 0975-9166, Volume 4, Issue 1, pp.-404.

Copyright: Copyright@2012 Angadi S.A., Vilas Naik and Ashwin Kumar. This is an open-access article distributed under the terms of the Creative Commons Atribution License, which permits unrestricted use, distribution, and reproduction in any medium, provided the original author and source are credited. 